ESAIM: COCV 21 (2015) 989-1001

DOI: $10.1051 / \mathrm{cocv} / 2014055$
ESAIM: Control, Optimisation and Calculus of Variations

www.esaim-cocv.org

\title{
EXISTENCE OF SOLUTIONS TO BILINEAR PROBLEMS WITH A CLOSED-LOOP CONTROL
}

\author{
JEAN-MARC CLÉRIN ${ }^{1}$
}

\begin{abstract}
Here we prove the existence of solutions to nonlinear differential inclusion problems with closed-loop control $\dot{z}+A(z)=B(u, z)+f, u \in U(t, z), z(0)=z^{0}$ where the operator $B$ is bilinear with respect to the control $u$ and the state $z$ in reflexive, separable Banach spaces denoted $Y$ and $V$, respectively. The operator $A$ is nonlinear in $V$, and given a positive real number $T$, the set-valued map $U$ is defined in $[0, T] \times V$. Without making any assumptions about the convexity of $U$, its values are taken to be non-empty closed, decomposable subsets of $Y$.
\end{abstract}

Mathematics Subject Classification. 34A60, 35A01, 35G20, 93B52.

Received June 13, 2013. Revised September 17, 2014.

Published online June 12, 2015.

\section{INTRODUCTION}

Given a positive real number $T$, we take the differential inclusion problems

$$
\left(E_{z^{0}, f}\right)\left\{\begin{array}{l}
\dot{z}+A(z)=B(u, z)+f \quad \text { a.e. } \quad t \in[0, T], \\
z(0)=z^{0} \text { and } u(t) \in U(t, z(t)) \quad \text { a.e. } t \in[0, T] .
\end{array}\right.
$$

to be nonlinear problems with closed-loop control. The operator $B$ is bilinear with respect to the control $u$ and the state $z$ in reflexive, separable Banach spaces denoted $Y$ and $V$, respectively. The operator $A$ is nonlinear in $V$. The set-valued map $U$ is defined in $[0, T] \times V$, and its values are obtained from the set of non-empty, closed subsets of $Y$. The initial state $z^{0}$ and $f$ are perturbation parameters. Setting $F(t, z)=[A(z)](t)+B(U(t, z), z)+f(t)$, the nonlinear closed loop control system $\left(E_{z^{0}, f}\right)$ can be reduced to the differential inclusion:

$$
\dot{z}(t) \in F(t, z(t)), z(0)=z^{0} .
$$

In this paper, the functional spaces $V$ and $Y$ under consideration are infinitely dimensional. It has been established (see Aubin and Frankowska [2]) that many results on differential inclusions obtained in the finite dimensional context can be extended to compact-valued maps in infinite dimensional Banach spaces. For information about the application of differential inclusions to the theory of control systems in finite dimensions, see Aubin and Cellina [1] and Clarke [7]. In order to prove the existence Theorem 4.2, which is the main result

Keywords and phrases. Nonlinear infinite system, differential inclusion, bilinear control, closed-loop control, feedback law, a priori estimates, Willett and Wong's lemma.

1 Université Paris-Sorbonne (Paris IV), 10 rue Molitor, 75016 Paris, France. Jean-Marc.Clerin@paris-sorbonne.fr 
obtained in this paper, we study the set-valued map $R: K \mapsto L^{1}(0, T ; Y)$, where $r \in(1 ; \infty]$, where $K$ is a weakly compact subset of the space in which we seek solutions, defined by:

$$
R(z):=\left\{u \in L^{r}(0, T ; Y) \mid u(t) \in U(t, z(t)) \quad \text { a.e. } \quad t \in[0, T]\right\}
$$

The lower semicontinuity of $R$ results from that of $U$, but the values of $R$ are generally neither compact, nor convex. The compactness hypothesis is too strong for the differential inclusion problem $\left(E_{z^{0}, f}\right)$ studied here. In line with Michael's continuous selection theorem (see Michael [16], Thm. 3.2") the set-valued maps are assumed to be lower semicontinuous maps and their values are taken to be convex in order to be able to make use of their compactness in the weak sense. If a subset of a reflexive Banach space is closed, bounded and convex, then it will be weakly compact ( $c f$. Brézis [5], Cor. III.19). Here again the convex hypothesis is too strong for the present purposes. However, the values of $R$ are decomposable into $L^{1}(0, T ; Y)$.

Definition 1.1. A subset $\mathscr{P}$ of $L^{1}(0, T ; Y)$ is called a decomposable subset if for all $u$ and $v$ belonging to $\mathscr{P}$ and any Lebesgue measurable subset $\mathscr{A}$ included in $[0, T]$, any function taking the value $u(t)$ when $t \in \mathscr{A}$ or the value $v(t)$ when $t \in[0, T] \backslash \mathscr{A}$ will belong to $\mathscr{P}$.

Since this property is a substitute for the convexity in the present case, it enables us to use selection theorems in infinite dimensional spaces. Fryszkowski has established the existence of a continuous selection in [11] in the case of a lower semicontinuous set-valued map of this kind with decomposable values defined in a compact metric space (namely, the segment $[0, T]$ in the present case). Bressan and Colombo have extended this result to set-valued maps in which these properties are defined in part of a separable metric space (see [4]).

The second important point worth noting in this paper is that, writing $\left(E_{z^{0}, f}\right)$ instead of $(1.1)$ enables us to focus on the bilinear control problems underlying the closed-loop problems. We previously established $(c f$. Clérin [8], Thm 1, and see Rem. 2, p. 123, on the advantage of dealing with consider the bilinear operator separately) that the bilinear control problem

$$
\left(\mathscr{E}_{z^{0}, u, f}\right)\left\{\begin{array}{l}
\dot{z}+A z=B(u, z)+f \quad \text { a.e. } \quad t \in[0, T] \\
z(0)=z^{0} \quad \text { a.e. } \quad t \in[0, T] .
\end{array}\right.
$$

is well posed in the sense of Hadamard. In other words, under specific assumptions, the above equation has a solution $z_{z^{0}, u, f}$, this solution is unique and it depends continuously on the parameter $z^{0}, u$ and $f$. The bilinear nature of our problems makes it possible to establish specific a priori state estimates, which will be useful for studying the differential inclusion problems $\left(E_{z^{0}, f}\right)$. If the initial condition and the disturbance are sufficiently small in appropriate norms (see the condition $(\mathscr{C})$ and the corresponding comment 2 below), then, a kind of Gronwall's lemma, the Willett and Wong's lemma (cf. [19]) give a priori state estimates (3.1)-(3.3), which are uniform with respect to admissible controls. These estimates are appropriate for using a fixed point theorem for the subsequent proof of existence.

This paper is organized as follows. The principles underlying Definition 4.1 of a solution $z$ for $\left(\mathscr{E}_{z^{0}, f}\right)$ are introduced in the suitable functional framework of a Gelfand triplet (see [12]). The assumptions $(\mathscr{H})$ are then listed. In Section 3, we establish the a priori state estimates (3.1)-(3.3). The condition $(\mathscr{C})$ applying to all the parameters, the initial state $z^{0}$, the disturbance $f$, and $T$, enables us to use the Willett and Wong's Lemma 3.2. In Section 4, we define a solution $(z, u)$ for $\left(E_{z^{0}, f}\right)$ and we write the main result of the paper, namely Theorem 4.2. The proof of this theorem is given in Section 5, where the existence of solutions $(z, u)$ to the problems $\left(E_{z^{0}, f}\right)$ is established using Schauder's fixed point theorem, and proved using Castaing's measurable selection theorem (see [6]) as well as Fryszkowski's continuous selection theorem (see [11]). In Section 6, we present two examples of nonlinear models in which the above result applies. 


\section{Setting of the Problems}

\subsection{Functional framework}

Let $H$ be a separable Hilbert space and let $V$ be a dense subspace of $H$ which has the structure of a reflexive, separable Banach space. We need to identify $H$ with its dual $H^{*}$, and the injections below are assumed to be continuous and dense:

$$
V \hookrightarrow H \hookrightarrow V^{*} .
$$

A triplet of this kind is called a Gelfand triplet (see [12]). The norms on $H, V$ and $V^{*}$ are denoted |.|, ||.\| and $\|\cdot\|_{*}$, respectively. There exist two positive constants $C$ and $C^{*}$ such that

$$
\frac{1}{C^{*}}\|\cdot\|_{*} \leq|\cdot| \leq C\|\cdot\| \text {. }
$$

The scalar product on $H$ is denoted $(.,$.$) and \langle., .\rangle_{V^{*} \times V}$ is abbreviated as $\langle.,$.$\rangle . Compatibility between the two$ products is required:

$$
(., .)=\langle., .\rangle_{\mid H \times V} \cdot
$$

For $p$ belonging to $(1, \infty)$, we take $L^{p}(0, T ; V)$ to denote all classes of strongly measurable maps $v:[0, T] \rightarrow V$, which are integrable in the sense of Bochner (see [3], p. 49), such that

$$
\int_{0}^{T}\|v(t)\|^{p} \mathrm{~d} t<\infty
$$

This gives a Banach space equipped with the norm

$$
\|v\|_{p}:=\left(\int_{0}^{T}\|v(t)\|^{p} \mathrm{~d} t\right)^{1 / p} .
$$

If there is any ambiguity about the norm, we will have to specify the notation; for instance, by writing $\|\cdot\|_{L^{p}(0, T ; H)}$. In the equation $\left(E_{z^{0}, f}\right)$ and $\left(\mathscr{E}_{z^{0}, u, f}\right)$, we use the notation $\dot{z}$ to express the derivative of $z$ with respect to time. The Bochner integral of an element $w$ of $L^{p}(0, T ; V)$ is

$$
\int_{(0, T)} w \in V
$$

The state space in which we seek possible solutions to $\left(\mathscr{E}_{z^{0}, u, f}\right)$ is

$$
W_{p p^{*}}:=\left\{z \in L^{p}(0, T ; V) \mid \exists w \in L^{p^{*}}\left(0, T ; V^{*}\right): z(t)=z^{0}+\int_{(0, t)} w \quad \text { a.e. } \quad t \in[0, T]\right\} .
$$

Thus, any solution is absolutely continuous and the initial condition $\left(\mathscr{E}_{z^{0}, u, f}\right)$ is: $w(0)=z^{0}$. The unique element $w$ of $\left(L^{p}(0, T ; V)\right)^{*}$ associated with $z$ is denoted $\dot{z}$ as usual. The control $u$ belongs to the space $L^{r}(0, T ; Y)$ where $r=\frac{p}{p-2}$ (we take $r=\infty$ when $p=2$ ). Unless specified otherwise, $Y$ is a reflexive, separable Banach space. This constraint on the control leads us to impose $p \in[2, \infty)$. We can then identify the dual of $L^{p}(0, T ; V)$ with $\left(L^{p}(0, T ; V)\right)^{*}$, where $p^{*}$ is the conjugate of $p(c f .[3]$, Thm. 3.1, p. 50). The set of states

$$
W_{p p^{*}}(0, T)=\left\{z \in L^{p}(0, T ; V) \mid \dot{z} \in L^{p^{*}}\left(0, T ; V^{*}\right)\right\},
$$

with the norm

$$
\|z\|_{W_{p p^{*}(0, T)}}:=\left(\|z\|_{p}^{2}+\|\dot{z}\|_{p^{*}}^{2}\right)^{1 / 2}
$$

is therefore a reflexive, separable Banach space that is injected continuously into $C(0, T ; H)$. In other words, every element of $W_{p p^{*}}(0, T)$ has a unique continuous representative. When there is no ambiguity, we simply take $W$ to denote the space $W_{p p^{*}}(0, T)$. 


\subsection{Assumptions $(\mathscr{H})$}

Here we list the assumptions used to show that $\left(\mathscr{E}_{z^{0}, u, f}\right)$ is well-posed in the functional framework described above and those relating to the set-valued map $U$.

$\left(H_{p}\right) p \in[2, \infty)$.

$\left(H_{z^{0}}\right) z^{0} \in H$.

$\left(H_{f}\right) f \in L^{p^{*}}(0, T ; H)$.

$\left(H_{A}\right) \quad A:[0, T] \times V \rightarrow V^{*}$

(1) $\forall v \in V, A(., v):[0, T] \longrightarrow V^{*}$ is measurable.

(2) $A(t,):. V \longrightarrow V^{*}$ is bounded for almost all $t \in[0, T]$. More specifically, noting $s:=\frac{p}{p-1-\alpha}$,

$$
\exists \alpha \in[0, p-1], \exists a_{1} \in L^{2}\left(0, T ; \mathbb{R}^{+}\right), \exists a_{2} \in L^{s}\left(0, T ; \mathbb{R}^{+}\right)
$$

such that

$$
\|A(t, v)\|_{*} \leq a_{1}(t)+a_{2}(t)\|v\|^{\alpha} \quad \text { a.e. } \quad t \in[0, T], \forall v \in V .
$$

(We cannot take $\alpha$ to be strictly greater than $p-1$, otherwise $s$ would be negative.)

(3) The operator $A(t,$.$) is p$-coercive for almost all values of $t \in[0, T]$ :

$$
\exists K>0: \forall v \in V,\langle A(t, v), v\rangle \geq K\|v\|^{p} .
$$

(4) The operator $A(t,$.$) is monotonic for almost all t \in[0, T]$ :

$$
\left\langle A\left(t, v_{1}\right)-A\left(t, v_{2}\right), v_{1}-v_{2}\right\rangle \geq 0, \quad \forall v_{1}, v_{2} \in V .
$$

(5) For all $v_{1}$ and $v_{2}$ in $V$, and for almost all $t \in[0, T]$, the operator $A$ is hemicontinuous; i.e.

$$
\lambda \in \mathbb{R}^{+} \longmapsto\left\langle A\left(t, v_{1}+\lambda v_{2}\right), v_{2}\right\rangle
$$

is continuous.

$\left(H_{u}\right) u \in L^{r}(0, T ; Y)$ where $r=\frac{p}{p-2}$ and $Y$ is a reflexive, separable Banach space.

$\left(H_{B}\right) B:[0, T] \times Y \times V \longrightarrow H$

(1) $\forall u \in Y, \forall v \in V, B(., u, v):[0, T] \longrightarrow H$ is measurable.

(2) $\forall v \in V, B(t, ., v) \in \mathscr{L}(Y, V)$ for almost all $t \in[0, T]$.

(3) $\forall u \in Y, B(t, u,.) \in \mathscr{L}(V)$ for almost all $t \in[0, T]$.

(4) $\exists b>0, \forall u \in Y, \forall v \in V:|B(t, u, v)| \leq b\|u\|_{Y}|v|$ for almost all $t \in[0, T]$.

(5) We assume the hemicontinuity of $B(t, u,$.$) . For all v_{1}$ and $v_{2}$ in $V$, for all $u$ in $Y$ and for almost all $t$ in $[0, T]$,

$$
\lambda \in[0 ; \infty) \longmapsto\left\langle B\left(t, u, v_{1}+\lambda v_{2}\right), v_{2}\right\rangle
$$

is continuous.

$\left(H_{U}\right)$ The set-valued map $U$ is defined on $[0, T] \times H$, and its values are obtained from the set of non-empty, closed subsets of $Y$ such that:

(1) The graph of $U$ belongs to $\mathscr{L} \otimes \mathscr{B}(H) \otimes \mathscr{B}(Y)$ where $\mathscr{L}$ is the Lebesgue tribe on $[0, T], \mathscr{B}(H)$ and $\mathscr{B}(Y)$ are the Borel tribes of $H$ and $Y$, respectively.

(2) $U(t,$.$) is lower semicontinuous for almost all t \in[0, T]$.

(3) $\exists \gamma \in[0 ; \infty), \exists c_{1} \in[0 ; \infty), \exists c_{2} \in(0 ; \infty), \forall v \in H$ :

$$
|U(t, v)|:=\sup \left\{\|u\|_{Y}: u \in U(t, v)\right\} \leq c_{1}+c_{2}|v|^{\gamma} \quad \text { a.e. } \quad t \in[0, T] .
$$




\section{Comments:}

(1) The assumption $\left(H_{p}\right)$ depends on $\left(H_{u}\right)$ because we must take the positivity of $r$ into account. When using the Galerkin method, the monotony and the hemicontinuity $\left(H_{A}\right)(4)-(5)$ and $\left(H_{B}\right)(5)$ are useful means of ensuring the continuity of the restrictions of these operators to spaces with finite dimensions. Coercivity $\left(H_{A}\right)(3)$ can be used to establish a priori estimates. In this article, operator $B$ is required to take its values in $H$. The validity of this hypothesis $\left(H_{B}\right)(4)$ is proved in the case of our examples. But another inequality is adopted with the bilinear operator $(u \cdot \nabla) z$ (see [9]). Lastly, the assumptions $\left(H_{z^{0}}\right),\left(H_{f}\right)\left(H_{A}\right)(1-2)$ and $\left(H_{B}\right)(1-4)$ can make the problem $\left(\mathscr{E}_{z^{0}, u, f}\right)$ meaningful in suitable functional spaces. The following two propositions justify the choice of spaces made in the previous section. The differential equation $\left(\mathscr{E}_{z^{0}, u, f}\right)$ is written in terms of $L^{p^{*}}\left(0, T ; V^{*}\right)$ and the hypothesis $\left(H_{z^{0}}\right)$ requires the initial state to belong to $H$.

(2) The exponent $\alpha$ featuring in $\left(H_{A}\right)(2)$ is generally assumed to be $1-p$ (see Lions [15], Thm. 1.2).

(3) In the study of nonlinear differential inclusion problems presented in [10], instead of assumptions $\left(H_{B}\right)(2)$ and $\left(H_{U}(3)\right)$ we find the inequalities

$$
|B(t, u, v)| \leq b\left(\|u\|_{Y}+|v|^{2 / p^{*}}\right)
$$

and

$$
|U(t, v)| \leq c_{2}\left(1+|v|^{2 / p^{*}}\right)
$$

which are not present in the bilinear system under investigation here.

\subsection{Choice of spaces}

We verify that our fuctional framework is meaningful for the statement of the problems $\left(\mathscr{E}_{z^{0}, u, f}\right)$ and $\left(E_{z^{0}, u, f}\right)$.

Proposition 2.1. Let $\left(H_{p}\right)$ and $\left(H_{A}\right)(1)-(2)$ hold for all $v$ in $L^{p}(0, T ; V)$, then

$$
A(., v(.)) \in L^{p^{*}}\left(V^{*}\right) \text {. }
$$

Proof. Start with the case where $\alpha \in(0, p-1)$. Given that

$$
\|A(., v(.))\|_{*} \leq a_{1}(.)+a_{2}(.)\|v(.)\|^{\alpha}
$$

and using the fact that $a_{1}$ is $p^{*}$-Lebesgue integrable, we only need to prove that $\left(a_{2}(.)\|v(.)\|^{\alpha}\right)^{p^{*}}$ is integrable. Apply Young's inequality:

$$
\left(a_{2}(.)\|v(.)\|^{\alpha}\right)^{p^{*}} \leq \frac{p^{*}}{s} a_{2}(.)^{s}+\frac{\alpha p^{*}}{p}\|v(.)\|^{p}
$$

where $a_{2}^{s}$ and $\|v\|^{p}$ are assumed to be integrable. If $\alpha$ is equal to zero, then $s$ will be equal to $p^{*}$ and if $\alpha$ is equal to $p-1$, then $s$ will be infinite. In both cases, we will also obtain the expected result.

Proposition 2.2. Assume $\left(H_{p}\right)$ and $\left(H_{B}\right)(1)-(4)$ for all $u \in L^{r}(0, T ; Y)$ and for all $v \in L^{p}(0, T ; V)$ :

$$
B(., u(.), v(.)) \in L^{p^{*}}(0, T ; H) .
$$

Proof.

$$
\int_{0}^{T}|B(t, u(t), v(t))|^{p^{*}} \mathrm{~d} t \leq \int_{0}^{T} \beta\|u(t)\|_{Y}^{p^{*}}|v(t)|^{p^{*}} \mathrm{~d} t .
$$

Taking $q$ to be the conjugate of $p-1$, we check that $p^{*} q=r$ and $p^{*}(p-1)=p$. We apply the Hölder inequality to $u \in L^{s}(0, T ; Y)$ and $v \in L^{p}(0, T ; V)$.

$$
\int_{0}^{T}|B(t, u(t), v(t))|^{p^{*}} \mathrm{~d} t \leq b\left(\int_{0}^{T}\|u(t)\|_{Y}^{p^{*} q} \mathrm{~d} t\right)^{1 / q}\left(\int_{0}^{T}|v(t)|^{p^{*}(p-1)} \mathrm{d} t\right)^{1 /(p-1)} .
$$

Therefore

$$
\|B(., u(.), v(.))\|_{L^{p^{*}}(0, T ; H)} \leq b C\|u\|_{r}\|v\|_{p} .
$$




\section{A priori state estimates And the COndition $(\mathscr{C})$}

\subsection{A priori state estimates}

Lemma 3.1. Let $\left(H_{p}\right),\left(H_{z^{0}}\right),\left(H_{f}\right),\left(H_{A}\right)(1),\left(H_{B}\right)$ and $\left(H_{U}\right)$ hold, if $A$ is positive, i.e.

$$
\forall v \in V,\langle A(t, v), v\rangle \geq 0 \quad \text { a.e. } \quad t \in[0, T]
$$

and if the condition

$$
\left(\left|z^{0}\right|^{2}+\|f\|_{L^{2}(0, T ; H)}^{2}\right)^{-\gamma / 2}-\frac{2 b c_{2}}{2 b c_{1}+1}\left(\mathrm{e}^{\left(b c_{1}+1 / 2\right) \gamma T}-1\right)>0
$$

holds when $\gamma>0$, then there exists a constant $M_{1}$ such that for any solution $(z, u)$ of $\left(E_{z^{0}, f}\right)$ :

$$
\|z\|_{C(0, T ; H)} \leq M_{1} \text {. }
$$

In addition, if we assume $\left(H_{A}(2)\right.$ and (3), then the constants $M_{2}$ and $M_{3}$ exist and are such that for any solution $(z, u)$ of $\left(E_{z^{0}, f}\right)$ :

$$
\begin{aligned}
\|z\|_{L^{p}(0, T ; V)} & \leq M_{2} \\
\|\dot{z}\|_{L^{p^{*}}\left(0, T ; V^{*}\right)} & \leq M_{3} .
\end{aligned}
$$

Comments:

(1) The constants $M_{1}, M_{2}$ and $M_{3}$ depends on $z^{0}$, on $f$, and on the given parameters, but not on the control $u$.

(2) We are now able to express more specifically the proviso "if the initial condition and the disturbance are sufficiently small", by writing:

$$
\sqrt{\left|z^{0}\right|^{2}+\|f\|_{L^{2}(0, T ; H)}^{2}}<\left[\frac{2 b c_{2}}{2 b c_{1}+1}\left(\mathrm{e}^{\left(b c_{1}+1 / 2\right) \gamma T}-1\right)\right]^{-1 / \gamma} .
$$

(3) On the one hand, given a positive real number $T$, the condition $(\mathscr{C})$ will hold true "if the initial condition and the disturbance are sufficiently small", and on the other hand, given an initial condition $z^{0}$ and a disturbance $f$, the condition $(\mathscr{C})$ will hold true for all $T \in(0 ; \bar{T})$, where we write

$$
\bar{T}=\frac{2 b c_{1}+1}{2 \gamma} \log \left[1+\frac{2 b c_{1}+1}{2 b c_{2}}\left(\left|z^{0}\right|^{2}+\|f\|_{L^{2}(0, T ; H)}^{2}\right)^{-\gamma / 2}\right]
$$

when $\gamma>0$ and $\bar{T}=\infty$ otherwise. If the initial condition and the disturbance are equal to zero then $\bar{T}$ will be infinite, and the condition $(\mathscr{C})$ will obviously be true.

\subsection{Proof}

Let us assume the existence of a state $z$ and a control $u$ such that $(z, u)$ is a solution of the equation $\left(E_{z^{0}, f}\right)$ where $z^{0}$ and $f$ are an initial state and a disturbance which satisfy the assumptions $\left(H_{z^{0}}\right)$ and $\left(H_{f}\right)$, respectively. For almost all $t$ belonging to $[0, T]$, the equality below holds.

$$
\langle\dot{z}(t), z(t)\rangle+\langle A(t, z(t)), z(t)\rangle=\langle B(t, u(t), z(t)), z(t)\rangle+\langle f(t), z(t)\rangle .
$$

The positivity of $A$, assumptions $\left(H_{B}\right),\left(H_{U}\right)$ and the compatibility of $(,$.$) with \langle., .\rangle_{\mid H \times V}$ make it possible to establish the following inequalities, for almost all $t \in[0, T]$.

$$
\begin{aligned}
& \frac{1}{2} \frac{\mathrm{d}}{\mathrm{d} t}|z(t)|^{2} \leq b\|u(t)\|_{Y}|z(t)|^{2}+|f(t) \| z(t)| \\
& \frac{1}{2} \frac{\mathrm{d}}{\mathrm{d} t}|z(t)|^{2} \leq b\left(c_{1}+c_{2}|z(t)|^{\gamma}\right)|z(t)|^{2}+\frac{1}{2}|f(t)|^{2}+\frac{1}{2}|z(t)|^{2} .
\end{aligned}
$$


Hence

$$
|z(t)|^{2} \leq\left|z_{0}\right|^{2}+\|f\|_{L^{2}(0, T ; H)}^{2}+2 b c_{2} \int_{0}^{t}\left(|z(s)|^{2}\right)^{1+\gamma / 2} \mathrm{~d} s+\left(2 b c_{1}+1\right) \int_{0}^{t}|z(s)|^{2} \mathrm{~d} s .
$$

Apply the inequality of the Gronwall type developed by Willett and Wong in [19].

Lemma 3.2. Let $T \in(0, \infty), c \in[0, \infty)$ and $h \in[0, \infty)$. Consider two integrable functions $x:[0, T] \rightarrow[0, \infty)$ and $y:[0, T] \rightarrow[0, \infty)$ and a Lebesgue measurable function $F:[0, T] \rightarrow[0, \infty)$ such that:

(1) $x F^{h}$ and $y F$ are integrable on $[0, T]$,

(2) $F(t) \leq c+\int_{0}^{t} y(s) F(s) \mathrm{d} s+\int_{0}^{t} x(s) F^{h}(s) \mathrm{d} s \quad$ a.e. $\quad t \in[0, T]$.

Then for almost all $t \in[0, T]$ :

(i) if $h \in\left[0,1\left[\right.\right.$ then $F(t) \leq \mathrm{e}^{\int_{0}^{t} y(s) \mathrm{d} s}\left(c^{1-h}+(1-h) \int_{0}^{t} x(s) \exp \left[(h-1) \int_{0}^{s} y(\theta) \mathrm{d} \theta\right] \mathrm{d} s\right)^{\frac{1}{1-h}}$,

(ii) if $h=1$ then $F(t) \leq c \mathrm{e}_{0}^{t} y(s) \mathrm{d} s+\int_{0}^{t} x(s) \mathrm{d} s$,

(iii) if $h \in(1, \infty)$ then the inequality (i) is also satisfied under the hypothesis:

$$
c^{1-h}+(1-h) \int_{0}^{T} x(s) \exp \left[(h-1) \int_{0}^{s} y(\theta) \mathrm{d} \theta\right] \mathrm{d} s>0 .
$$

Denote $F:=|z(.)|^{2}, h:=1+\gamma / 2, c:=\left|z^{0}\right|^{2}+\|f\|_{L^{2}(0, T ; H)}^{2}, x:=2 b c_{2}$ and $y:=2 b c_{1}+1$. If $\gamma>0$, then the condition (iii) can be written:

$$
\left(\left|z^{0}\right|^{2}+\|f\|_{L^{2}(0, T ; H)}^{2}\right)^{-\gamma / 2}-(\gamma / 2) \int_{0}^{T} 2 b c_{2} \exp \left[(\gamma / 2) \int_{0}^{s}\left(2 b c_{1}+1\right) \mathrm{d} \theta\right] \mathrm{d} s>0 .
$$

By hypothesis:

$$
\left(\left|z^{0}\right|^{2}+\|f\|_{L^{2}(0, T ; H)}^{2}\right)^{-\gamma / 2}-\frac{2 b c_{2}}{2 b c_{1}+1}\left(\mathrm{e}^{\left(b c_{1}+1 / 2\right) \gamma T}-1\right)>0 .
$$

Applying Willet and Wong's lemma, for almost all $t \in[0, T]$ gives:

$$
|z(t)|^{2} \leq \mathrm{e}^{T\left(1+2 b c_{1}\right)}\left[\left(\left|z^{0}\right|^{2}+\|f\|_{L^{2}(0, T ; H)}^{2}\right)^{-\gamma / 2}-\frac{2 b c_{2}}{2 b c_{1}+1}\left(\mathrm{e}^{\left(b c_{1}+1 / 2\right) \gamma T}-1\right)\right]^{-2 / \gamma} .
$$

We deduce the first estimate a priori

$$
\|z\|_{C(0, T ; H)} \leq M_{1}
$$

where

$$
M_{1}:=\mathrm{e}^{T\left(b c_{1}+1 / 2\right)}\left[\left(\left|z^{0}\right|^{2}+\|f\|_{L^{2}(0, T ; H)}^{2}\right)^{-\gamma / 2}-\frac{2 b c_{2}}{2 b c_{1}+1}\left(\mathrm{e}^{\left(b c_{1}+1 / 2\right) \gamma T}-1\right)\right]^{-1 / \gamma} .
$$

Let us consider the special case where $\gamma=0$.

$$
\begin{array}{r}
|z(t)|^{2} \leq\left|z^{0}\right|^{2}+\|f\|_{L^{2}(0, T ; H)}^{2}+\left(2 b\left(c_{1}+c_{2}\right)+1\right) \int_{0}^{t}|z(s)|^{2} \mathrm{~d} s \quad \text { a.e. } \quad t \in[0, T] \\
|z(t)|^{2} \leq\left(\left|z^{0}\right|^{2}+\|f\|_{L^{2}(0, T ; H)}^{2}\right) \mathrm{e}^{\left(2 b\left(c_{1}+c_{2}\right)+1\right) T} \quad \text { a.e. } t \in[0, T] .
\end{array}
$$

The Bellman lemma directly yields the estimate (3.1). 
We now have to prove the second a priori estimate (3.2), assuming the p-coercivity $\left(H_{A}\right)(3)$.

$$
\begin{aligned}
\frac{1}{2} \frac{\mathrm{d}}{\mathrm{d} t}|z(t)|^{2}+K\|z(t)\|^{p} & \leq b\|u(t)\|_{Y}|z(t)|^{2}+|f(t) \| z(t)| \quad \text { a.e. } \quad t \in[0, T] \\
K \int_{0}^{T}\|z(t)\|^{p} \mathrm{~d} t & \leq \frac{1}{2}\left|z^{0}\right|^{2}+b \int_{0}^{T}\|u(t)\|_{Y}|z(t)|^{2} \mathrm{~d} t+\int_{0}^{T}|f(t) \| z(t)| \mathrm{d} t \quad \text { a.e. } \quad t \in[0, T] \\
K \int_{0}^{T}\|z(t)\|^{p} \mathrm{~d} t & \leq \frac{1}{2}\left|z^{0}\right|^{2}+b T M_{1}^{2}\left(c_{1}+c_{2} M_{1}^{\gamma}\right)+M_{1}\|f\|_{1} \quad \text { a.e. } \quad t \in[0, T] .
\end{aligned}
$$

Therefore

$$
\|z\|_{L^{p}(0, T ; V)} \leq M_{2}
$$

where

$$
M_{2}:=\frac{1}{K^{1 / p}}\left(\frac{1}{2}\left|z^{0}\right|^{2}+b T M_{1}^{2}\left(c_{1}+c_{2} M_{1}^{\gamma}\right)+M_{1}\|f\|_{1}\right)^{1 / p} .
$$

The proof of the third a priori estimate (3.3) is based on the hypothesis $\left(H_{A}\right)(2)$.

$$
\|\dot{z}(t)\|_{*} \leq \| A\left(t, z(t) \|_{*}+C(|B(t, u(t), z(t))|+|f(t)|) \quad \text { a.e. } \quad t \in[0, T]\right.
$$

where $C$ is a constant such that: $\|\cdot\|_{*} \leq C|\cdot|$.

$$
\left.\|\dot{z}(t)\|_{*} \leq a_{1}(t)+a_{2}(t)\|z(t)\|^{\alpha}+C b M_{1}\left(c_{1}+c_{2} M_{1}^{\gamma}\right)+|f(t)|\right) .
$$

We take $a_{3}():.=C b M_{1}\left(c_{1}+c_{2} M_{1}^{\gamma}\right)+a_{1}()+.|f()$.$| .$

$$
\begin{aligned}
\|\dot{z}(t)\|_{*} & \leq a_{3}(t)+a_{2}(t)\|z(t)\|^{\alpha} \\
\|\dot{z}(t)\|_{*}^{p^{*}} & \leq 2^{p^{*}-1}\left[\left|a_{3}(t)\right|^{p^{*}}+\left|a_{2}(t)\right|^{p^{*}}\|z(t)\|^{\alpha p^{*}}\right] \\
\|\dot{z}\|_{p^{*}}^{p^{*}} & \leq 2^{p^{*}-1}\left[\int_{0}^{T}\left|a_{3}(t)\right|^{p^{*}} \mathrm{~d} t+\int_{0}^{T}\left|a_{2}(t)\right|^{p^{*}}\|z(t)\|^{\alpha p^{*}} \mathrm{~d} t\right] .
\end{aligned}
$$

Based on $\left(H_{A}\right)(2)$ and $\left(H_{f}\right), a_{3}$ belongs to $L^{p^{*}}\left(\mathbb{R}^{+}\right)$. Young's inequality ensures that $\left(a_{2}(.)\|z(.)\|^{\alpha}\right)^{p^{*}}$ is summable and

$$
\int_{0}^{T}\left|a_{2}(t)\right|^{p^{*}}\|z(t)\|^{\alpha p^{*}} \mathrm{~d} t \leq \frac{p^{*}}{s}\left\|a_{2}\right\|_{s}^{s}+\frac{\alpha p^{*}}{p}\|z\|_{p}^{p}
$$

Using (3.2), we obtain the a priori estimate (3.3)

$$
\|\dot{z}\|_{p^{*}} \leq M_{3}
$$

where

$$
\left.M_{3}:=\left\{2^{p^{*}-1}\left\|a_{3}\right\|_{L^{p^{*}\left(0, T ; \mathbb{R}^{+}\right)}}^{p^{*}}+\frac{p^{*}}{s}\left\|a_{2}\right\|_{L^{s}\left(0, T ; \mathbb{R}^{+}\right)}^{s}+\frac{\alpha p^{*}}{p} M_{2}^{p}\right]\right\}^{1 / p^{*}} .
$$

\section{The EXISTENCE RESUlt}

Definition 4.1. The initial state $z^{0}$ and the perturbation $f$ are adopted in the spaces $H$ and $L^{p^{*}}(0, T ; H)$, respectively. A solution for $\left(E_{z^{0}, f}\right)$ will be $(z, u) \in W_{p p^{*}}(0, T) \times L^{r}(0, T ; Y)$ so that $\left(E_{z^{0}, f}\right)$ is satisfied.

Theorem 4.2. Assuming that $(\mathscr{C})$ is satisfied and that $V$ has been injected compactly into $H$, if the assumptions $(\mathscr{H})$ hold, then the problem $\left(E_{z^{0}, f}\right)$ has a solution in $W_{p p^{*}}(0, T) \times L^{r}(0, T ; Y)$. 


\section{Proof of existence theorem}

Let $u \in L^{r}(0, T ; Y)$ be a control. Given $z^{0} \in H, f \in L^{2}(0, T ; H)$ and $T>0$ such that the hypothesis $(\mathscr{H})$ is satisfied, the state equation $\left(E_{u}\right)$ below is no longer governed by a control law. Its unknown is $z$ and it is well posed in the sense of Hadamard (see [8]).

$$
\left(E_{u}\right)\left\{\begin{array}{l}
\dot{z}(t)+A(t, z(t))=B(t, u(t), z(t))+f(t) \quad \text { a.e. } \quad t \in[0, T] \\
z(0)=z^{0}
\end{array}\right.
$$

Let us consider

$$
\begin{aligned}
\xi:\left(L^{r}(0, T ; Y),\|\cdot\|_{r}\right) & \rightarrow\left(W, \sigma\left(W, W^{*}\right)\right) \\
u & \mapsto \quad z_{u}
\end{aligned}
$$

where $z_{u}$ is the single solution of $\left(E_{u}\right)$.

Lemma 5.1. The map $\xi$ is continuous from $\left(L^{r}(0, T ; Y),\|\cdot\|_{r}\right)$ to $\left(W, \sigma\left(W, W^{*}\right)\right)$.

Proof. The map $\xi$ is locally Lipschitz $\left(c f\right.$. [8]), and therefore it is continuous from $\left(L^{r}(0, T ; Y),\|.\|_{r}\right)$ to $\left(W,\|\cdot\|_{W}\right)$.

Define

$$
\mathscr{V}:=\left\{u \in L^{r}(0, T ; Y):\|u\|_{r} \leq M\right\}
$$

where $M:=T^{1 / r}\left(c_{1}+c_{2} M_{1}^{\gamma}\right)$.

Comment: We have chosen $M$ such that for all solutions $(z, u)$ of $\left(E_{z^{0}, f}\right)$, the control $u$ belongs to $\mathscr{V}$.

Lemma 5.2. The set $K:=\overline{c o}[\xi(\mathscr{V})]$ is compact in $\left(W, \sigma\left(W, W^{*}\right)\right)$.

Here we take $\overline{c o}[\xi(\mathscr{V})]$ to denote the closed convex cone generated by $\xi(\mathscr{V})$.

Proof. The a priori estimates $\left(E_{2}\right)$ and $\left(E_{3}\right)$ make it possible to show that $K$ is a closed, convex bounded subset of the reflexive Banach space $\left(W,\|\cdot\|_{W}\right)$. It is therefore a subset of $\sigma\left(W, W^{*}\right)$-compact.

Let us now define the set-valued map $R$ from $K$ to the set of non-empty closed subsets of $L^{r}(0, T ; Y)$ :

$$
R(z):=\left\{u \in L^{r}(0, T ; Y): u(t) \in U(t, z(t)) \text { a.e. } t \in[0, T]\right\} .
$$

The subset $\mathscr{D} \subset L^{r}(0, T ; Y)$ is called decomposable if for all $u$ and all $v$ in $\mathscr{D}$ and for every Lebesgue measurable subset $A$ included in $[0, T]$,

$$
w_{A}(t):=\left\{\begin{array}{l}
u(t) \text { if } t \in A, \\
v(t) \text { if } t \in[0, T] \backslash A
\end{array}\right.
$$

belongs to $\mathscr{D}$.

Lemma 5.3. The values of the set-valued map $R$ are decomposable.

Proof. Given $z \in K$, for all $u$ and for all $v$ in $R(z)$ and for every Lebesgue measurable subset $A$ included in $[0, T]$ define:

$$
w_{A}(t):=\left\{\begin{array}{l}
u(t) \text { if } t \in A, \\
v(t) \text { if } t \in[0, T] \backslash A .
\end{array}\right.
$$

By construction, $\left.w_{A} t\right) \in\{u(t), v(t)\} \subset U(t, z(t))$ and $w_{A} \in R(z)$. Hence for all $z$ the value $R(z)$ is decomposable. 
We have now to prove the lower semicontinuity of $R$. The space $\left(K, \sigma\left(W, W^{*}\right)\right)$ is metrizable, for any closed subset $F$ included in $L^{r}(0, T ; Y)$ and for any sequence $\left(z_{n}\right)_{n \in \mathbb{N}^{*}} \sigma\left(W, W^{*}\right)$-converging to $z$ such that for all $n \in \mathbb{N}^{*}, R\left(z_{n}\right) \subset F$, we are going to prove that $R(z) \subset F$.

Lemma 5.4. Given $\bar{u} \in R(z)$, if $F$ is a closed subset included in $L^{r}(Y)$ and if $\left(z_{n}\right)_{n \in \mathbb{N}^{*}}$ is a sequence $\sigma\left(W, W^{*}\right)$ converging to $z$ such that for all $n \in \mathbb{N}^{*}, R\left(z_{n}\right) \subset F$, there exists a measurable function $u_{n}$ from $[0, T]$ to $Y$ such that

$$
\left\{\begin{array}{l}
u_{n} \in R\left(z_{n}\right), \\
\left\|u_{n}(t)-\bar{u}(t)\right\|_{Y} \leq \frac{1}{n}+\mathrm{d}\left(\bar{u}(t), U\left(t, z_{n}(t)\right)\right) \quad \text { a.e. } \quad t \in[0, T] .
\end{array}\right.
$$

Proof. Let us consider the set-valued map

$$
\Gamma_{n}(t):=\left\{u \in U\left(t, z_{n}(t)\right): \mathrm{d}\left(\bar{u}(t), U\left(t, z_{n}(t)\right)+\frac{1}{n} \geq\|u-\bar{u}(t)\|_{Y} \text { a.e. } t \in[0, T]\right\}\right.
$$

the graph $\Gamma_{n}$ of which is

$$
\operatorname{Gr}\left(\Gamma_{n}\right):=\left\{(t, u) \in[0, T] \times Y: u \in \Gamma_{n}(t)\right\}
$$

Define

$$
\varphi_{n}(t, u):=\mathrm{d}\left(\bar{u}(t), U\left(t, z_{n}(t)\right)+\frac{1}{n}-\|u-\bar{u}(t)\|_{Y}\right.
$$

The graph $\Gamma_{n}$ is:

$$
\operatorname{Gr}\left(\Gamma_{n}\right)=\left\{(t, u) \in[0, T] \times Y: \varphi_{n}(t, u) \geq 0 \quad \text { a.e. } \quad t \in[0, T]\right\} .
$$

Based on $\left(H_{U}\right)(1)$ and $(2), \varphi_{n}(t,$.$) is continuous and \varphi_{n}(., u)$ is measurable, $\varphi_{n}(.,$.$) and \operatorname{Gr}\left(\Gamma_{n}\right)$ are therefore also measurable. Any measurable section $u_{n}$ of $\Gamma_{n}$ (see [6]) satisfies the above statement.

Lemma 5.5. The set-valued map $R$ is lower semicontinuous from $\left(K, \sigma\left(W, W^{*}\right)\right)$ to $\left(L^{r}(0, T ; Y),\|\cdot\|_{r}\right)$.

Proof. The sequence $z_{n} \sigma\left(W, W^{*}\right)$-converges to $z$. Based on Simon's theorem (see [17]), we can extract a subsequence written in the same way, such that for almost all $t \in[0, T], z_{n}(t)$ converges to $z(t)$ in $H$. The lower semicontinuity of $U(t,$.$) makes it possible to deduce the upper semicontinuity of the map \zeta \mapsto \mathrm{d}(v, U(t, \zeta))$ for all $v \in Y$ and for almost all $t \in[0, T]$ (see [13], Prop. 2.26).

$$
0 \leq \limsup _{n \rightarrow \infty} \mathrm{d}\left(\bar{u}(t), U\left(t, z_{n}(t)\right)\right) \leq \mathrm{d}(\bar{u}(t), U(t, z(t)))
$$

where

$$
\mathrm{d}(\bar{u}(t), U(t, z(t)))=0 \quad \text { a.e. } \quad t \in[0, T] .
$$

Therefore, for almost all $t \in[0, T],\left(u_{n}(t)\right)_{n \in \mathbb{N}^{*}}$ converges strongly in $Y$ to $\bar{u}(t)$. Given that for $n$ of $\mathbb{N}^{*}$

$$
\left\|z_{n}\right\|_{C(0, T, H)} \leq M_{1}
$$

we have

$$
\left\|u_{n}(t)\right\|_{Y} \leq c_{1}+c_{2} M_{1}^{\gamma} \quad \text { a.e. } \quad t \in[0, T] .
$$

The proof of the convergence of this sequence in $L^{r}(0, T ; Y)$ is based on the Lebesgue dominated convergence theorem. Since $F$ is closed we deduce:

$$
\bar{u} \in F .
$$

In conclusion, $R$ is lower semicontinuous. 
The values of the set-valued map $R$ are decomposable and $R$ is lower semicontinuous, and hence the Fryszkowski continuous selection theorem ([11], Thm. 3.1) proves the existence of at least one function denoted $r: K \rightarrow \mathscr{V}$ which is continuous from $\left(W, \sigma\left(W, W^{*}\right)\right)$ to $\left(L^{1}(0, T ; Y),\|\cdot\|_{1}\right)$ and such that

$$
\forall z \in K, r(z)(t) \in U(t, z(t)) \text { a.e. } t \in[0, T] .
$$

The set-valued map $R$ is the selection values in $L^{r}(0, T ; Y)$ (included in $L^{1}(0, T ; Y)$ ). Therefore $r$ is continuous from $\left(W, \sigma\left(W, W^{*}\right)\right)$ to $\left(L^{r}(0, T ; Y),\|\cdot\|_{1}\right)$. Applying the fact that $\xi$ is continuous from $\left(L^{r}(0, T ; Y),\|\cdot\|_{1}\right)$ to $\left(W, \sigma\left(W, W^{*}\right)\right)$ and writing these two functions in the same way, we deduce that $\xi \circ r: K \rightarrow K$ is $\sigma\left(W, W^{*}\right)$ continuous from the convex and weakly compact $K$ subset to itself. By applying the fixed point theorem of Schauder to $\xi \circ r: K \rightarrow K$, we prove the existence of a function $\bar{z} \in K$ such that $\xi \circ r(\bar{z})=\bar{z}$, i.e. $\bar{z}$ is the only solution associated with the control $r(\bar{z})$ in $U(., \bar{z}()$.$) and (\bar{z}, r(\bar{z}))$ is a solution of $\left(E_{z^{0}, f}\right)$.

\section{EXAMPLES}

\subsection{Nonlinear model for the mechanical deformation of ice}

In the field of continuum mechanics, elastic deformation is characterized by its reversibility. Linear elasticity involves small deformations where stretching and twisting are proportional to the force and torque exerted, respectively. However, in the case of larger deformations, there exist more suitable nonlinear elastic models. We take the example of the mechanical deformation of a thin sheet of ice. The p-Laplacian operator is a strongly nonlinear operator with $p>2$ (see Lions [15]). Let $\Omega$ be a bounded and connected subset of $\mathbb{R}^{2}$, with a regular boundary $\partial \Omega$, and let $T$ be positive. Control is distributed over the domain space $\Omega$. The deformation (in terms of a displacement and an angle) is the state of the system denoted $z$. The three parameters of interest here are the initial condition $z^{0}$, the mechanical control $u$ exerted on all or part of the surface and the disturbance term $f$ resulting from the combined effects of external forces (e.g. the weight of the plate, the irradiation or thermal variations). This problem can be modeled using the following PDE:

$$
\left(P D E_{z^{0}, u, f}\right)\left\{\begin{array}{l}
z_{t}(t, x, y)-\operatorname{div}\left(|\nabla z(t, x, y)|^{p-2} \nabla z(t, x, y)\right) \\
=\Sigma_{i=1}^{2} u_{i}(t) z_{i}(t, x, y)+f(t, x, y) \quad \text { in }(0, T) \times \Omega \\
z(t, x, y)=0 \quad \text { on }(0, T) \times \partial \Omega \\
z(0, x, y)=z^{0}(x, y) \quad \text { in } \Omega .
\end{array}\right.
$$

The control drives the damping or the increase in this deformation. One type of control consists in setting the masses on the surface of the ice (see Trentin and Guyaner [18]). Other models involve the use of dynamic controls when the structure is subjected to a harmonic excitation focusing on specific areas or an acoustic wave inducing vibration $\left(c f\right.$. Yu [20]). The Gelfand triplet $W_{0}^{1, p}(\Omega) \hookrightarrow L^{2}(\Omega) \hookrightarrow W^{-1, p}(\Omega)$ adapted to constraints on the boundary $\partial \Omega$ can be used to reduce this problem to a differential equation where the unknown (still denoted by $z$ ) depends only on time. Here, $p^{*}:=\frac{p}{p-1}$, the set $\left.W^{1, p} \Omega\right)$ contains the elements of $L^{2}(\Omega)$ whose partial derivatives also belong to $L^{2}(\Omega)$, and $V:=W_{0}^{1, p}(\Omega)$ can be identified with the set of elements $W^{1, p}(\Omega)$ which vanish almost everywhere on the boundary $\partial \Omega$. The choice of the space $V$ depends on the behavior of the state at the boundary of the domain $\Omega$. With $v \in V$, the p-Laplacian operator is defined by

$$
\Delta_{p} v:=-\operatorname{div}\left(|\nabla v|^{p-2} \nabla v\right)
$$

and we then study the conditions on which the stability of the differential equation depends in this functional framework:

$$
\left\{\begin{array}{l}
\dot{z}(t)-\Delta_{p} z(t)=u(t) z(t)+f(t) \quad \text { a.e. } \quad t \in[0, T] \\
z(0)=z^{0} \in L^{2}(\Omega) .
\end{array}\right.
$$

To preserve the elasticity in this problem, it may be useful to impose a box constraint on the controls. From the theoretical point of view, a wide range of possible constraints can be imposed on closed loops. 


\subsection{Model chain reaction with diffusion}

We now develop a reaction model for a reaction process where the diffusion and convection processes are controlled by introducing chemical reagents which either act as catalysts or slow down a chain reaction (see Khapalov [14]). This is also a model for heat exchange, the control and the fluid velocity. (We can also envisage modeling a mass exchange occuring during the diffusion process.) Let $\Omega$ be a bounded and connected subset of $\mathbb{R}^{n}(n \in\{1,2,3\})$, with a regular boundary $\partial \Omega$, and let $T$ be positive. The state equation is:

$$
\left(E R C_{z^{0}, u, f}\right)\left\{\begin{array}{l}
z_{t}(t, x)-\Delta z(t, x)=u(t, x) z(t, x)+f(t, x) \text { in }(0, T) \times \Omega \\
z(t, x, y)=0 \quad \text { on }(0, T) \times \partial \Omega \\
z(0, x)=z_{0}(x) \in L^{2}(\Omega) .
\end{array}\right.
$$

In the case of diffusion alone, the control $u$ and $f$ are equal to zero. The reaction equation $(E R C)$ is:

$$
\left\{\begin{array}{l}
z_{t}(t, x)=u(t, x) z(t, x) \quad \text { in }(0, T) \times \Omega \\
z(t, x, y)=0 \quad \text { on }(0, T) \times \partial \Omega \\
z(0, x)=z_{0}(x) \in L^{2}(\Omega)
\end{array}\right.
$$

The Gelfand triplet $H_{0}^{2}(\Omega) \hookrightarrow L^{2}(\Omega) \hookrightarrow H^{-2}(\Omega)$ is adapted to constraints on the boundary $\partial \Omega$, and makes it possible to reduce this problem to a differential equation where the unknown (still denoted by $z$ ) depends only on time.

$$
\dot{z}(t)-\Delta z(t)=u(t) z(t)+f(t) \quad \text { a.e. } \quad t \in(0, T)
$$

The initial condition is chosen in $L^{2}(\Omega)$ :

$$
z(0)=z^{0}
$$

To give just one example, since the state is the mass of a reaction product, the control can be taken to be the concentration of a catalyst introduced during this reaction. To control this closed-loop reaction, we introduce an amount of catalyst depending on the mass produced.

\section{REFERENCES}

[1] J.-P. Aubin and A. Cellina, Differential inclusions, Set-valued maps and viability theory. Vol. 264 of Grundlehren der Mathematischen Wissenschaften [Fundamental Principles of Mathematical Sciences]. Springer-Verlag, Berlin (1984).

[2] J.-P. Aubin and H. Frankowska, Set-valued analysis. Birkhäuser, Boston (2008).

[3] V. Barbu and Th. Precupanu, Convexity and optimization in Banach spaces. Vol. 10 of Math. Appl. (East European Series). D. Reidel Publishing Co., Dordrecht, romanian edition (1986).

[4] Alberto Bressan and Giovanni Colombo, Extensions and selections of maps with decomposable values. Studia Math. 90 (1988) 69-86.

[5] H. Brézis, Analyse fonctionnelle. Théorie et applications. [Theory and applications]. Collection Mathématiques Appliquées pour la Maîtrise. [Collection of Applied Mathematics for the Master's Degree]. Masson, Paris (1983).

[6] C. Castaing and M. Valadier, Convex analysis and measurable multifunctions. Vol. 580 of Lect. Notes Math. Springer-Verlag, Berlin (1977).

[7] F. Clarke, Optimization and Nonsmooth Analysis. Wiley-Interscience, New York (1983).

[8] J.-M. Clérin, Équations d'état bien posées en contrôle bilinéaire (well-posed state equations in bilinear control). Rev. Roumaine Math. Pures Appl. 56 (2011) 115-136.

[9] J.-M. Clérin, Analyse de sensibilité d'un problème de contrôle optimal bilinéaire. Ann. Mat. Blaise Pascal 19 (2012) $177-196$.

[10] A. Fiacca, N.S. Papageorgiou and F. Papalini, On the existence of optimal controls for nonlinear infinite-dimensional systems. Czechoslovak Math. J. 48 (1998) 291-312.

[11] A. Fryszkowski, Continuous selections for a class of nonconvex multivalued maps. Studia Math. 76 (1983) $163-174$.

[12] I.M. Gel'fand and N.Ya. Vilenkin, Generalized functions. Vol. 4 of Applications of harmonic analysis. Translated by Amiel Feinstein. Academic Press, New York (1964).

[13] S. Hu and N.S. Papageorgiou, Handbook of Multivalued Analysis: Theory. Vol. 419 of Math. Appl. Springer (1997).

[14] A.Y. Khapalov, Controllability of the semilinear parabolic equation governed by a multiplicative control in the reaction term: a qualitative approach. SIAM J. Control Optim. 41 (2003) 1886-1900. 
[15] J.-L. Lions, Quelques méthodes de résolution des problèmes aux limites non linéaires. Dunod Gauthier-Villars (1969).

[16] E. Michael, Continuous selections 1. Ann. Math. 63 (1956) 361-382.

[17] J. Simon, Compact sets in the space $L^{p}(0, T ; B)$. Ann. Mat. Pura Appl. 146 (1987) 65-96.

[18] D. Trentin and J.-L. Guyaner, Vibration of a master plate with attached masses using modal sampling method. J. Acoust. Soc. Am. 96 (1994) 235-245.

[19] D. Willett and J.S.W. Wong, On the discrete analogues of some generalizations of Gronwall's inequality. Monatsh. Math. 69 (1965) 362-367.

[20] Y.-Y. Yu, Vibrations of elastic plates. Springer (1995). 